\title{
Application of Fuzzy Analytic Hierarchy Process \\ to Group Decision Making
}

\author{
- HU ZHAOGUANG \\ Electric Power Research Institute \\ Qinghe, Beijing 100085, CHINA
}

\begin{abstract}
It is known that group decision making is very important, especially in the early stage of power development. In China, due to the uncoordinated distribution of the industrial structure and the excessively rapid growth of electricity consuming equipment, the nationwide shortage of electric power has become a conspicuous problem in the economic and social development. Therefore, China must adopt effective measures to accelerate the development of the power industry and adjust its industrial structure. In this paper, we develop a new method for group decision making and a decision support system for generation expansion planning.
\end{abstract}

\section{Introduction}

Generation expansion planning is a necessary and important work in the early stage of the development of a power system. Its main task is to determine the type of new units and the capacities which must be installed each year during the planning period in order to economically meet the need of power consumption as predicted by the load forecast within the limits of given constraints. This problem is usually modelled as a mathematical programing problem. However, besides the objective of the minimization of the total input during the whole planning period, there are other objectives such as generation reliabilities, environmental policies and social requirements that ought to be considered. of course, the single objective programming formulation is easier than the multi-objective one. However, the former fails to take many factors into consideration. In addition, social requirements policies are difficult to be modeled mathematically because they are represented by nonquantifiable factors. To quantify these factors, we use fuzzy sets.

Another key problem is that decisions must be made by groups. Generation expansion planning needs to consider thermal, hydrological, gas and nuclear power policies. Therefore, there are a lot of opinions from experts, many of which are contradictory. 
It is very difficult to reach a compromise in opinions and it usualiy requires many meetings without results.

In this case, group decision making in factor space [2] has been discussed by using the fuzzy AHP [3], and the problem can be solved automatically by a computer after a man-machine dialogue. To provide easy control by the experts on the computer optimization procedure, a decision support system (MGD) has been built to help support decision makers in the selection of optimal decisions.

In the following section, we shall discuss how to express nonquantified factors in decision making as fuzzy sets, and how to model group decision making in multiobjective generation expansion planning program and to explain the structure of MGD. A numerical example is given at the end of the paper.

\section{Nonquantified Decision Factors Expressed By Fuzzy sets}

The nonquantifiable factors in generation expansion planning are policies, social requirements and macroscopic factors.

\section{i) Policies : $P=\{\mathrm{p} 1, \mathrm{p} 2, \mathrm{p} 3, \mathrm{p} 4, \ldots\}$}

where

pl : preference of developing medium-sized hydro-electric power station.

p2 : to build more thermal power stations near coal mines.

p3 : to promote local investment.

p4 : to refrain from using oil as fuel for power stations.

\section{ii) Social Factors : $\mathrm{S}=\{\mathrm{s} 1, \mathrm{~s} 2, \mathrm{~s} 3, \mathrm{~s} 4, \ldots\}$}

where

s1 : social benefits

s2 : sources of local funds for power station.

s3 : to enhance flourishing of industry and agriculture in the area near power station.

s4 : strategic importance of the power station.

iii) Macroscopic factors : $M=\{m 1, m 2, m 3, \ldots\}$

where

$m 1$ : to promote the benignant cycle of power system development to be economically auto-progressive.

$\mathrm{m} 2$ : the effect on the industrial structure.

m3 : the effect on the structure of power sources.

The importance of the above factors can be quantitatively 
expressed by R-fuzzy sets of type 2 [1]. Let set $T=\{S, P, M\}$ and the fuzzy set $W$ in $T$ represent importance in $T$ expressed as a membership function. For instance,

$$
u_{w}(P)=\frac{0.5}{0.4}+\frac{1.0}{0.5}+\frac{0.5}{0.6}
$$

means that element $\mathrm{P}$ is comparatively important, i.e. the element $P$ in $T$ is comparatively important. While in $P$ we can define a fuzzy set having a fuzzy grade of

$$
\mathrm{u}_{\mathrm{w}}(\mathrm{p} 4)=\frac{0.5}{0.7}+\frac{1.0}{0.8}+\frac{0.5}{0.9}
$$

which means that the factor $\mathrm{p} 4$ is very well satisfied. The expressions (1) and (2) can be shown as Figure 1 . Here the nonquantitative factors in decision making are quantified by using

- fuzzy sets. Thus, it is easy to handle the above factors in the mathematical model.

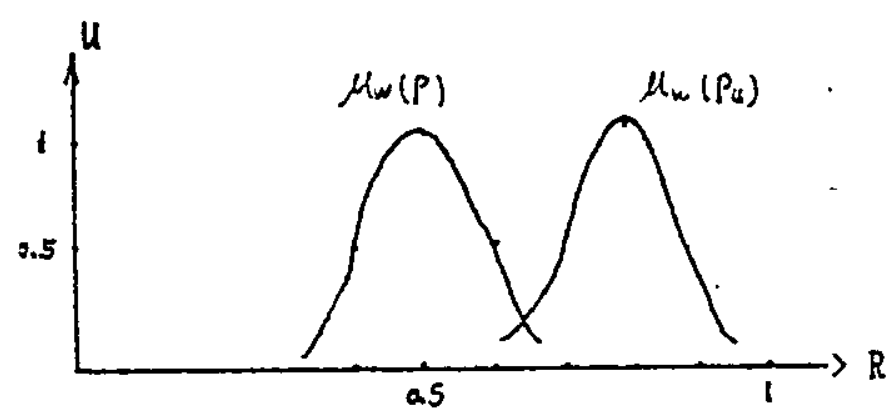

Fig. 1

Figure 1

\section{Group Decision Making}

To discuss group decision making in the factor space, we introduce the concept of factor space briefly. The concept of factor space is generated from comparisons. Comparing aims to find out what is the difference among objects. But, different objects 
are comparable only when they have some factor in common. There is a well known saying in chinese, "wind and crow can not be mentioned in the same breath". They are different and there is no meaning in their comparison. Therefore, comparing needs a factor combining different objects into a common comparable aspect.

Factor $f_{1}$ is called subfactor of factor $f_{2}$ if any state of $f_{2}$ determines a state of $f_{1}$. For example, $f_{1}=$ length of rectangle, $f_{2}=$ shape of rectangle. The length of a rectangle is determined by its shape, so that $f_{1}$ is a subfactor of $f_{2}$. obviously, we have:

$$
\begin{aligned}
x_{f 2}=\left\{\begin{array}{l}
(x, y) \mid x, y \text { are the length and width of a rectangle } \\
\text { respectively. }\}
\end{array}\right. \\
x_{f 1}=\{x \mid x \text { is the length of a rectangle }\}
\end{aligned}
$$

and the state space of the subfactor is a subspace of the state space of that factor. We can give this definition in a serious mathematical approach but omit to state them in detail here.

Factor $f$ is called the intersection of factors $f 1$ and $f 2$, if $f$ is a subfactor of both $f l$ and $f 2$, and any other common subfactor of $f I$ and $f 2$ is a subfactor of $f$. We have:

$$
f=f I \cap f 2 \text {. }
$$

A factor $f$ is called simple if it has no subfactors. The set of simple factors is called the fundamental family of factors, denoted by $S$. If there exists the simple family of factors, then any factor $f$ can be viewed as a subset of $s$, so that the set of all factors in a problem can be viewed as the power of $s$, i.e.

$$
F=P(S)=\{A \mid A \subseteq S\} \text {. }
$$

The power of $S$ is a Boolean Algebra. We have:

$$
\begin{aligned}
x_{f} & =\prod_{g \subset f} x_{g} \\
x_{f q} & =\prod_{g \subset f\}} X_{g}
\end{aligned}
$$

Here we can write the mathematical definition of factor space as follows:

Definition 1. A factor space is a family of sets $\left\{X_{f}\right\}$ (f $\subseteq F$ ) where $F$ is Boolean algebra $F=(F,,, c)$ satisfying $x_{0}=\{\#\}:\left(\forall T \subseteq F: t_{1}, t_{2} \in T, t_{1} \neq t_{2} \Rightarrow t_{1} \cap t_{2}=\theta\right) \Rightarrow X_{V} \in T=\prod_{S \in T} X_{S}$ 
Where 0 and 1 are the smallest element and largest element of $F$ respectively.

Now, we give a new concept fah as follows.

Definition 2. Let $T=\left\{t \mid \forall t_{i}, t_{j} \in F\right.$ and $\left.t_{i} n t_{j}=\varnothing\right\}$ in the factor space $\left(X_{f}\right)(f \in F)$, then $S: X_{t} \rightarrow X_{i}, t \in T$. Let $A_{t}$ be a fuzzy set in $X_{t}$ and $W$ is a fuzzy set in $T$, then the intersection of $S\left[A_{t}\right]$ and $S[W]$ is also a fuzzy set in $X$, i.e., $z=S\left[A_{t}\right] \cdot S[W] . z$ is called fah.

Theorem 1. Let $T=\{t\} \forall t_{i}, t_{j} \in F$ and $\left.t_{i} \cap t_{j}=0\right\}$ be $a$ in the factor space $\left\{X_{f}\right\}(f \in F)$. If the fuzzy set $A_{t}$ in $X_{t}(t \in T)$ is convex and the fuzzy set $W$ in $T$ is also convex, then $Z=S\left[A_{t}\right] \cdot S[W]$ is convex. Proof: (For the proof, see [4])

Now we can discuss decision making in the space of factors. Its Boolean algebra is shown in Figure 2 .

\{Policies, Social factors, Macroscopic factors\}
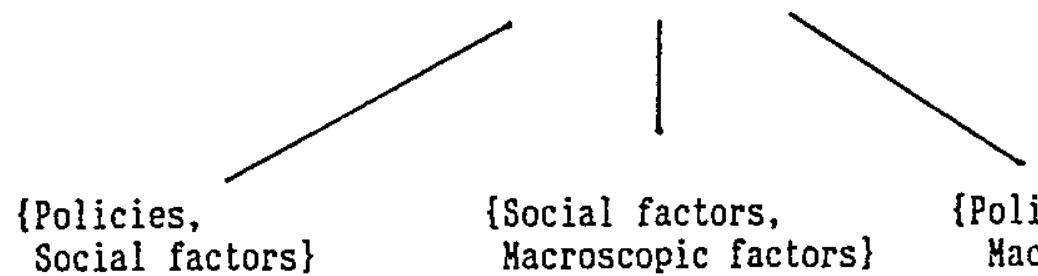

Social factors

\{Social factors, Macroscopic factors

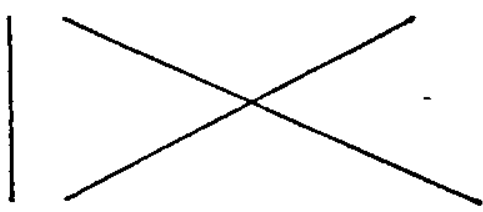

\{Social factors\}

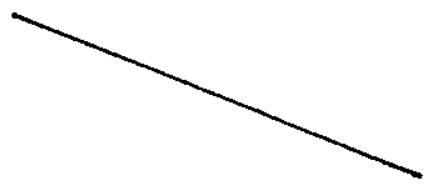

\{Policies\}

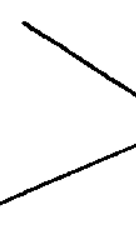

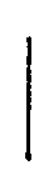

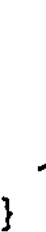

\{Policies, Hacroscopic

; factors\}

$\{0\}$

Fig.2 Boolean Algebra

It can be simply expressed as

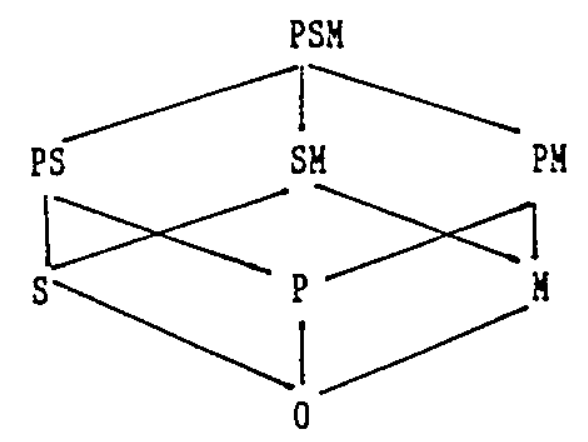

Fig. 3 
since $S \cap P=0, P \cap M=\varnothing$ and $S \cap M=0$, ten $T=\{S, P, M\}$. If there are $n$ factors/criteria and $m$ experts/decision makers, the group decision making can be expressed as:
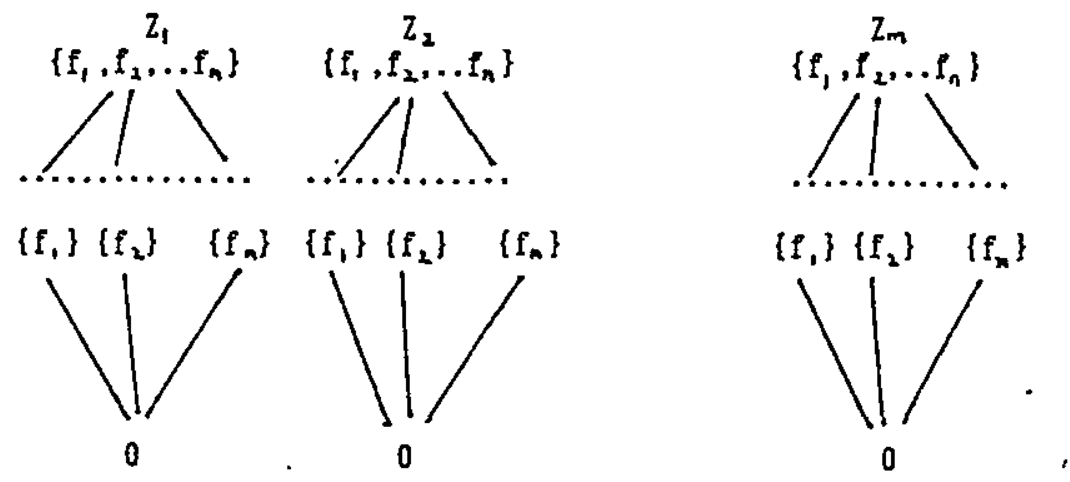

We can see that there a $m$ fah $z_{i}(i=1,2, \ldots, m)$, the result of group decision making is given by:

$$
\mathrm{Z}=\mathrm{n} \mathrm{z}_{\mathbf{i}}
$$

It can be easily shown that $z$ is convex.

\section{Multiobjective Model of Generation Expansion Planning}

Besides input investment as an objective in conventional planning methods, another objective of semi-structured decision making is also introduced in order to consider nonquantifiable factors in our decision making. Then, the planning model has two objective functions for generation expansion planning as follows [2]:

$$
\begin{aligned}
& \min \mathrm{Z1}=\mathrm{AX}+\mathrm{BY} \\
& \max \mathrm{Z} 2=\mathrm{FX}
\end{aligned}
$$

subject to:

$$
\begin{aligned}
\mathrm{CX}+\mathrm{DY} & <\mathrm{E} \\
\mathrm{X} & >0 \\
\mathrm{Y} & =0 \text { or } 1 .
\end{aligned}
$$

where

$X$ : additional capacities of power plant for the year [MKW] .

$Y$ : hydro-electric station installed or not.

$A$ : unit cost of plant [MYUAN/MKW]

$B$ : investment of the dam for hydro-electric station [MYUAN]

$F$ : grade of evaluation for nonquantitative factors related to $x$ in semi-structured decision making. 
Problem (3) can be solved as follows [3]:

(1) Separate formula (3) into two parts with one objective in each, $\min \mathrm{Z} 1=\mathrm{AX}+\mathrm{BY}$

subject to:

$$
\begin{aligned}
\mathrm{CX}+\mathrm{DY} & <\mathrm{E} \\
\mathrm{X} & >0 \\
\mathrm{Y} & =0
\end{aligned}
$$

and

$$
\max \mathrm{Z2}=\mathrm{FX}
$$

subject to:

$$
\begin{aligned}
C X+D Y & <\mathrm{E} \\
\mathrm{X} & >0 \\
\mathrm{Y} & =0 \text { or } 1
\end{aligned}
$$

Let $\left(z_{1}^{\prime}, x_{1}\right)$ and $\left(z_{2}^{\prime}, x_{2}\right)$ be the solutions of (4) and (5), respectively.

(2) The membership function can be written as

$$
U I=\left\{\begin{array}{cl}
\frac{-Z I^{\prime}+Z I(X)}{Z I-Z I^{\prime}} & Z I<Z I(X) \\
0 & Z I^{\prime} \leq Z I(X) \leq Z I \\
& Z I(X) \leq Z I^{\prime}
\end{array}\right.
$$$$
U 2=\left\{\begin{array}{cc}
\frac{Z 2(X)-Z 2^{\prime}}{Z 2-Z 2^{\prime}} & Z 2<Z 2(X) \\
0 & Z 2^{\prime} \leq Z 2(X) \leq Z 2 \\
Z 2(X) \leq Z 2^{\prime}
\end{array}\right.
$$

(3) We can solve for $\lambda$ from the following set of equations:

$$
\max \lambda
$$

subject to:

$$
\begin{aligned}
& \lambda \leq u_{1} \\
& \lambda \leq u_{2} \\
& C X+D Y \leq E \\
& X \geq 0 \\
& Y \in\{0,1\}
\end{aligned}
$$

(4) Haying obtained $\lambda$ from (6), the following set of equations can be solved: 


$$
\min s=s_{1}+s_{2}
$$

subject to:

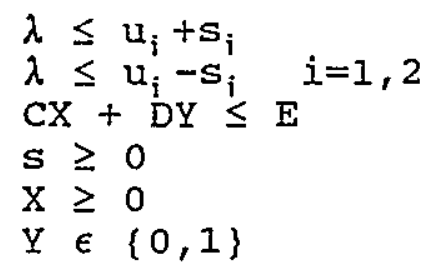

Substituting $x$ from (7) into (3), $z_{1}$ and $z_{2}$ can be obtained.

5. Decision Support System for Generation Expansion Planning

The structure of the decision support system for generation expansion planning (MGD) is shown in Figure 4.

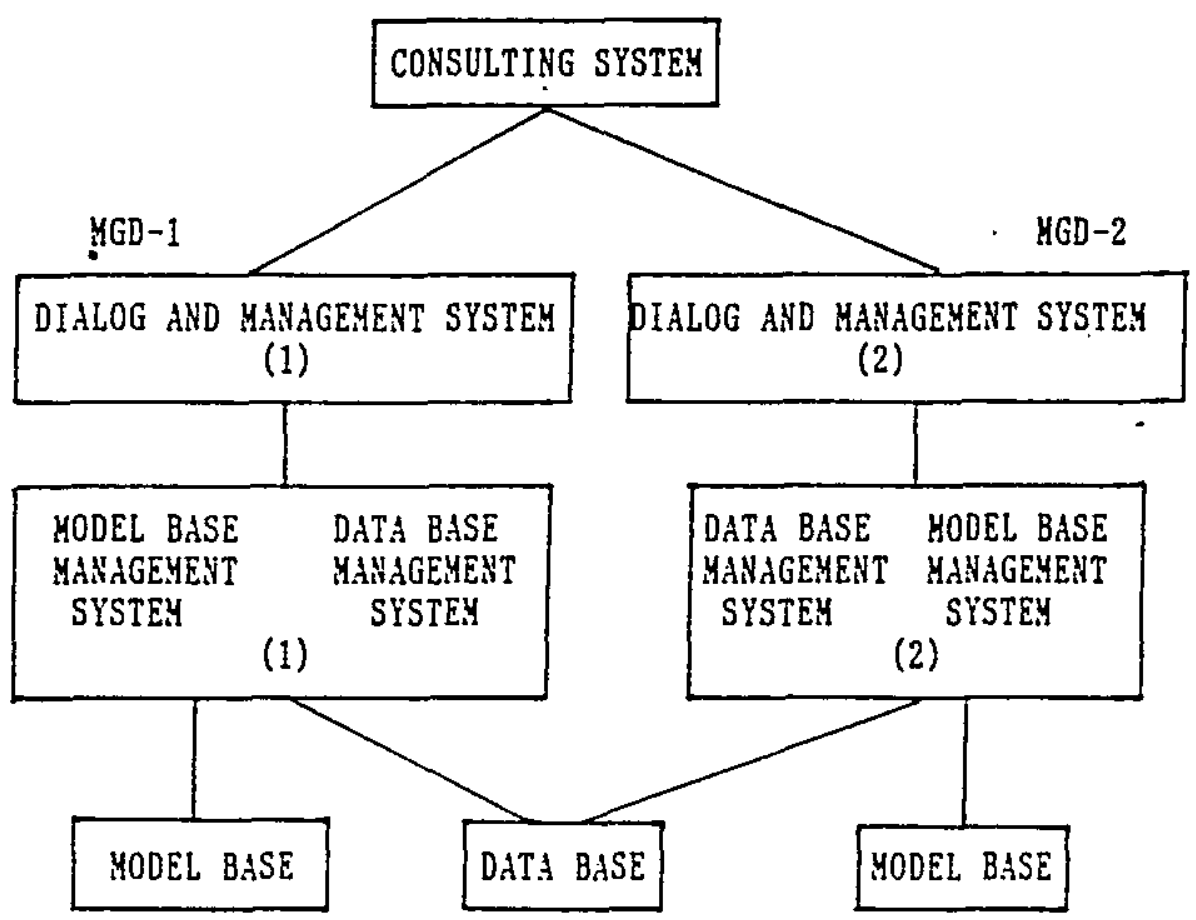

Figure 4. Structure of MGD

In the man-machine interactive system, the consulting system tells the user the necessary information about what MGD is going to do, i.e. generation expansion planning with one or two objectives. It also tells the user the main method of generation expansion 
planning. Then, it asks the user what he wants to do. If the user wants to do generation expansion planning with one objective, it turns to subsystem MGD-2.

MGD-I includes the dialogue system, a model management system and a data management system. The dialogue system is a management system for one objective. It includes menus of graphical representations and numerical tables that can be selected by the user. The management system model can generate a mathematical model. Since it is only for generation expansion planning with one objective, i.e. objective function is the minimization of the total input during the planning period, its main function is to set restrictions according to the user's need (or practice). Then the data management system provides input data from the data base according to the needs of the model.

MGD-2 is a system for generation expansion planning with two objectives, $i . e$. besides the minimization of the total input as an objective function, another one for nonquantified factors in semistructured decision making as mentioned above is included. It is formed through dialogue between the user and the computer. The user must answer a series of questions asked by the computer. The semi-structured factors can be quantified by using fuzzy sets and an objective function can be formed. Model management system "can build up the planning model, and the data management system can provide all kinds of data needed by the model.

There is a method-base providing a library of mathematical programming software. It includes IP software, MILP software, fuzzy analysis of hierarchical process software and fuzzy multiobjective mixed integer programing software.

MGD can support generation expansion planning as follows:

(1) It can rapidly provide optimized alternatives for generation expansion planning not only with one objective but also with two objectives. It can also provide exact total input during the planning period corresponding to this optimized alternative, reliability LOLP, capacities of new units, operating cost and so on.

(2) MGD can give the main factors restricting generation 
development by its analytic abilities quantitatively. For instance, is it possible to transmit power from area $A$ to area B? If it is possible, how much does it cost? If it is not possible, what is its main restrictions and why? (and so on) Therefore, it can support decision makers to analyze the situation of real problems from several sides.

(3) It can help decision makers to perform sensitivity analysis.

(4) It can not only promote the use LOLP in reliability but it can also help decision makers to analyze what kinds of units are better at different levels of LOLP.

(5) MGD can help decision makers to analyze the effect of alternatives in generation expansion planning after changing some nonquantifiable factors in semi-structured decision making. Therefore, it can support decision makers to select the best alternatives to meet power development.

(6) The most important is that MGD can use nonquantifiable factors which are difficult to be considered by conventional methods in generation expansion planning model. After receiving expert's experiences, it combines the mathematical model and human expert's experiences in the computer. Therefore, it can only bring the advantages of each into play while avoiding the individual disadvantages.

\section{Numerical Example}

To demonstrate the usefulness of MGD, we give below a case study [4]:

In a power system, the peak load grows at an annual rate of 7 percent. An estimate of it in the year 2000 will be $54,782 \mathrm{MW}$. The system has existing units with total capacity of $68,720 \mathrm{MW}$. The user wants to know what kinds of units and what capacity is needed during the planning period 2001-2015. The power of the units selected is $200 \mathrm{MW}, 300 \mathrm{MW}, 600 \mathrm{MW}$ and $800 \mathrm{MW}$. The user also wants to know if two hydroelectric power stations W1 and W2 should be instalied or not. According to MGD-1, the results is shown in Table 1 .

We can see that a 600MW unit will be the main unit during 2001-2015 and a 800MW unit will be the main unit after the year 
2008. Hydro-electric power station $W_{1}$ will be set in year 2015, and $W_{2}$ will not be needed during the planning period, $i . e . W_{1}$ is more economic that $W_{2}$.

However, some experts do not think so. They say that $W_{2}$ is worth considering because it will promote industry development. The social benefit of $W_{2}$ is greater than that of $W_{1}$. Therefore, $W_{2}$ will be more preferable that $W_{1}$.

Table 1. The results of Planning

\begin{tabular}{|c|c|c|c|c|c|c|c|}
\hline & & & & & & & MW \\
\hline YEAR & $<200$ & 300 & 600 & 800 & $\mathrm{~W}_{1}$ & $\mathrm{~W}_{2}$ & LOLP $<$ \\
\hline 2001 & 0 & 0 & 1414.9 & 0 & 0 & 0 & 0.1515 \\
\hline 2002 & & & 5349.3 & & & & 0.1093 \\
\hline 2003 & & & 5758.4 & & & & 0.0764 \\
\hline 2004 & & & 6075.8 & . & & & 0.0505 \\
\hline 2005 & & & 6555.9 & & & & 0.0329 \\
\hline 2006 & & & 7051.9 & & & & 0.0212 \\
\hline 2007 & & & 7431.9 & & & & 0.0129 \\
\hline 2008 & & & & 7192.7 & & & 0.0129 \\
\hline 2009 & & & & 7717.7 & & & 0.0129 \\
\hline 2010 & & & & 8089.4 & & & 0.0129 \\
\hline 2011 & & & & 8710.3 & & & 0.0129 \\
\hline 2012 & & & & 9352.9 & & & 0.0129 \\
\hline 2013 & & & & 9786.8 & & & 0.0129 \\
\hline 2014 & & & & 10561.2 & & & 0.0129. \\
\hline 2015 & & & $=$ & 9779. & 120. & & 0.0129 \\
\hline
\end{tabular}

other experts do not agree with them. They think that the area's economic development is not the concern of the electrical power company but that of the local government, and that... the objective of generation expansion planning is to minimize the total cost.

To reconcile these different viewpoints, we use MGD-2, to solve this problem, the result is shown in table 2 .

\section{Conclusions}

From the above discussions, we can draw the following conclusions:

(1) The group decision making used in fuzzy AHP, has been discussed in factor space. The result can be obtained from the meet of fah which is a useful concept defined in this paper. It can easily compromise the different opinions and is useful in practice. 
Table 2. The Planning Results With Expert's Viewpoints

\begin{tabular}{|c|c|c|c|c|c|c|c|}
\hline YEAR & $<200$ & 300 & 600 & 800 & $\mathrm{~W}_{1}$ & $\mathrm{~W}_{2}$ & LOLP< \\
\hline 2001 & 0 & 0 & $1414: .9$ & 0 & 0 & 0 & 0.1515 \\
\hline 2002 & & & 5349.3 & & & & 0.1093 \\
\hline 2003 & & & 0 & 6330.7 & & & 0.0764 \\
\hline 2004 & & & & 6689.2 & & & 0.0505 \\
\hline 2005 & & & & 7229.9 & & & 0.0329 \\
\hline 2006 & & & & 7790.8 & & & 0.0212 \\
\hline 2007 & & & & 8209.6 & & & 0.0129 \\
\hline 2008 & & & & 7177.3 & & & 0.0129 \\
\hline 2009 & & & & 7703.5 & & & 0.0129 \\
\hline 2010 & & & & 8068.5 & & & 0.0129 \\
\hline 2011 & & & & 8696.8 & & & 0.0129 \\
\hline 2012 & & & & 9343.9 & & & 0.0129 \\
\hline 2013 & & & & 9767.1 & & & 0.0129 \\
\hline 2014 & & & & 10549.2 & & & 0.0129 \\
\hline 2015 & & & & 8211.7 & 120 & 120 & 0.0129 \\
\hline
\end{tabular}

The best compromised alternative is easier to be accepted by experts of both sides.

(2) The mathematical model of generation expansion planning with two objectives can include more factors in the model such as nonquantified factors in semi-structured decision making which are difficult to handle by conventional methods. Therefore, it can help decision makers to consider more factors in generation expansion planning.

(3) A decision support system has been made for generation expansion planning, which is a first DSS in electric power systems in china. Human experiences can be expressed in the form of fuzzy sets through dialogue with the computer. It combines mathematical model and human expert's viewpoints to do the planning work, bringing both advantages into play. This is a new method in generation expansion planning.

(4) The structure of MGD simplifies decision making. Since all models are represented by separate independent modules, it is easy to modify any one of them and to add new ones to meet the new needs of the users.

(5) The more important advantage is that it can accept all different ideas of different domain experts. We can see that it is impossible to do so by using conventional models. 


\section{REFERENCES}

[1] T.L.Saaty, The Analytic Hierarchy Process, McGraw-Hill Company, 1980.

[2] Wang Peizhuang, Factor Space and Knowledge Representation

[3] Hu Zhaoguang, Fuzzy AHP and its Applications

[4] Hu . Zhaoguang, Decision Support System for Generation Expansion Planning, Thesis for Ph.D. 1989.

[5] Hu Zhaoguang, Application of Fuzzy Mathematical Programming to Electric Power Supply Allocation, Proceedings of International Symposium on Fuzzy Systems and Knowledge Engineering, p465, 1987. 